\title{
Dimensional crossover of the fundamental-measure functional for parallel hard cubes
}

\author{
José A. Cuesta and Yuri Martínez-Ratón \\ Grupo Interdisciplinar de Sistemas Complicados (GISC), \\ Departamento de Matemáticas, Escuela Politécnica Superior, Universidad Carlos III de Madrid, \\ c/ Butarque, 15, 28911 - Leganés, Madrid, Spain
}

\begin{abstract}
We present a regularization of the recently proposed fundamental-measure functional for a mixture of parallel hard cubes. The regularized functional is shown to have right dimensional crossovers to any smaller dimension, thus allowing to use it to study highly inhomogeneous phases (such as the solid phase). Furthermore, it is shown how the functional of the slightly more general model of parallel hard parallelepipeds can be obtained using the zero-dimensional functional as a generating functional. The multicomponent version of the latter system is also given, and it is suggested how to reformulate it as a restricted-orientation model for liquid crystals. Finally, the method is further extended to build a functional for a mixture of parallel hard cylinders.
\end{abstract}

PACS numbers: 61.20.Gy, 64.10.+h, 68.45.-v

Although nowadays the success of density functional theories to describe nonuniform fluids and their phase transitions is out of question, it is generally acknowledged that these theories are based on much to empiric assumptions to be considered a systematic tool to study liquids [1]. Nearly all of the available functionals propose a dependence on the density through nonlocal weights, whose choice rely upon approximations around the uniform fluid combined with heuristic arguments. Accordingly, they always need a good knowledge of the bulk structure and thermodynamics of the uniform fluid, which thus becomes an input of the functional. There is, however, a kind of functionals which do not follow this general framework and are constructed only from geometric features of the particles; these are the so called fundamental measure functionals (FMFs) [2, 近. Two important features distinguish FMFs from "classical" functionals: (1) the thermodynamics and structure of the uniform fluid (and even that of the nonuniform fluid) can be derived from them, instead of requiring it as an input, and (2) they achieve a dimensional reduction of the system by using strongly inhomogeneous density profiles [5,66. Actually these two properties are interlaced, as it will become clear in what follows.

The first FMF was proposed for a fluid of hard spheres (HS) [2,3], and it was obtained by assuming it to depend on a small set of geometrically-weighted densities (how many and which weights is dictated by the low density limit of the free-energy functional). The precise functional dependence still required an ad hoc scaledparticle assumption, but in turn, it yielded the PercusYevick (PY) free energy and direct correlation function (DCF), and allowed to compute further structural properties (such as the third order DCF [2]). The functional admits two-dimensional (2D) [7] as well as a onedimensional (1D) [2] extensions, the latter being the wellknown exact functional.

There is a feature the exact HS functional exhibits: a right dimensional crossover, or, in other words, the fact that the functional for $D-1$ dimensions must come out as a result of the one for $D$ dimensions when the density profile is a delta function along one of the coordinates. But this turns out to be a much to stringent requirement to be fulfilled by an approximate functional. Needless to say that functionals with this property will provide good descriptions of strongly inhomogeneous systems (e.g. fluids confined by walls). However, it would not be of great importance to describe bulk phases were it not because there is a special dimensional crossover any functional willing to exhibit a sensible solid phase should verify: the reduction to a 0 -dimensional (0D) system [5, 7, i.e. a cavity able to contain at most one particle (which clearly mimics the situation of a particle in a crystal lattice). None of the "classical" functionals verify any dimensional crossover [9], but the FMF for HS does some of them [5]; however, when trying to reduce from 3D to $0 \mathrm{D}$, some divergences appear that cause this functional not to be able to stabilize a solid phase. Although an heuristic modification of the functional eliminates those unphysical divergencies [5], it is very remarkable that the D-dimensional HS FMF, for any dimension, can be obtained from the exact $0 \mathrm{D}$ functional by requiring it to have a right dimensional crossover from $D$ to quasi- 0 dimensions [6] (according to Ref. [6], a quasi-0D system is provided by a density function consisting of a sum of deltas placed at points such that HS simultaneously placed at them overlap). The results turn out to be nonlocal functionals of the one-particle weighted densities, which can be further approximated by local ones without loosing their main dimensional crossovers. The moral of this is that the $0 \mathrm{D}$ functional seems to be the only thing one needs in order to make a FMF for a given system.

Recently, a FMF has been developed for a system of parallel hard cubes (PHC) [4]. Although such a system is rather unrealistic, it is far more suitable than the HS system to study the demixing transition of mixtures [4, 10], 
and so, it deserves consideration. On the other hand, on a fundamental viewpoint, having FMFs for systems other than HS may help to clarify the basic structure of such functionals in the aim of extending them to more general particle shapes (arbitrarily orientable, anisotropic particles, for instance, so as to study liquid-crystalline phases). In this letter we will show how the functional proposed in [4] can be regularized to have right dimensional crossovers, and this will make clear a simple relation between the FMF for PHC in different dimensions which will permit to derive them very easily from the 0D functional.

Any FMF expresses the excess (over the ideal) free energy of the system in terms of the local density $\rho(\mathbf{r})$ as

$$
F^{\mathrm{ex}}[\rho]=k_{B} T \int d \mathbf{r} \Phi^{(D)}\left(\left\{n_{\alpha}(\mathbf{r})\right\}\right),
$$

where $D$ is the dimension and the $n_{\alpha}(\mathbf{r})$ are a set of suitably chosen geometrically-weighted densities. According

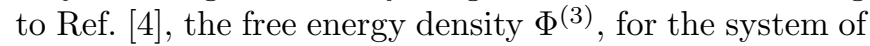
(3D) PHC, is given by $\Phi^{(3)}=\Phi_{1}^{(3)}+\Phi_{2}^{(3)}+\Phi_{3}^{(3)}$, with

$$
\begin{aligned}
& \Phi_{1}^{(3)}=-n_{0} \ln \left(1-n_{3}\right), \quad \Phi_{2}^{(3)}=\frac{\mathbf{n}_{1} \cdot \mathbf{n}_{2}}{1-n_{3}} \\
& \Phi_{3}^{(3)}=\frac{5 n_{2}^{3}-9 n_{2} \mathbf{n}_{2} \cdot \mathbf{n}_{2}}{54\left(1-n_{3}\right)^{2}} .
\end{aligned}
$$

Here $n_{\alpha} \equiv \rho \otimes \omega_{\alpha}$ and $\mathbf{n}_{\alpha} \equiv \rho \otimes \mathbf{w}_{\alpha}$, where $\otimes$ denotes a convolution. The weights $\omega_{\alpha}$ and $\mathbf{w}_{\alpha}$ are given by

$$
\begin{aligned}
\omega_{0}(\mathbf{r}) & =\zeta_{x} \zeta_{y} \zeta_{z}, \quad \omega_{3}(\mathbf{r})=\tau_{x} \tau_{y} \tau_{z} \\
\mathbf{w}_{1}(\mathbf{r}) & =\left(\tau_{x} \zeta_{y} \zeta_{z}, \zeta_{x} \tau_{y} \zeta_{z}, \zeta_{x} \zeta_{y} \tau_{z}\right) \\
\mathbf{w}_{2}(\mathbf{r}) & =\left(\zeta_{x} \tau_{y} \tau_{z}, \tau_{x} \zeta_{y} \tau_{z}, \tau_{x} \tau_{y} \zeta_{z}\right) \\
\omega_{1}(\mathbf{r}) & =\mathbf{u} \cdot \mathbf{w}_{1}(\mathbf{r}), \omega_{2}(\mathbf{r})=\mathbf{u} \cdot \mathbf{w}_{2}(\mathbf{r})
\end{aligned}
$$

with $\tau_{x}=\Theta(\sigma / 2-|x|), \zeta_{x}=(1 / 2) \delta(\sigma / 2-|x|)(\sigma$ being the edge-length of the cubes), and $\mathbf{u}=(1,1,1)$. Notice the different normalization in the definition of the weighted densities with respect to that of Ref. [4].

As mentioned in [4], the 2D functional can be obtained in a similar way; in this case the weights are given by

$$
\begin{aligned}
\omega_{0}(\mathbf{r})=\zeta_{x} \zeta_{y}, & \omega_{2}(\mathbf{r})=\tau_{x} \tau_{y}, \\
\mathbf{w}_{1}(\mathbf{r})=\left(\tau_{x} \zeta_{y}, \zeta_{x} \tau_{y}\right), & \omega_{1}(\mathbf{r})=\mathbf{u} \cdot \mathbf{w}_{1}(\mathbf{r}),
\end{aligned}
$$

with $\mathbf{u}=(1,1)$, and $\Phi^{(2)}=\Phi_{1}^{(2)}+\Phi_{2}^{(2)}$, with

$$
\Phi_{1}^{(2)}=-n_{0} \ln \left(1-n_{2}\right), \quad \Phi_{2}^{(2)}=\frac{n_{1}^{2}-\mathbf{n}_{1} \cdot \mathbf{n}_{1}}{2\left(1-n_{2}\right)} .
$$

Of course, the 1D functional is the same as that of HS (i.e. the exact one) because in this case both, HS and $\mathrm{PHC}$, reduce to segments on a line. Therefore, $\Phi^{(1)}=\Phi_{1}^{(1)}=-n_{0} \ln \left(1-n_{1}\right)$, where $\omega_{0}(\mathbf{r})=\zeta_{x}$ and $\omega_{1}(\mathbf{r})=\tau_{x}$.

Let us now consider the $1 \mathrm{D}$ to $0 \mathrm{D}$ dimensional crossover. To this purpose let us define the class of quasi$0 \mathrm{D}$ densities as the set of functions with support in the interval $(-\sigma / 2, \sigma / 2)$ (the fact that this interval is centered around zero is completely irrelevant and it is only assumed for simplicity). The densities $\rho(x)=\delta(x)$ and $\rho(x)=\sum_{i} \eta_{i} \delta\left(x-x_{i}\right)$, with $-\sigma / 2<x_{i}<\sigma / 2, \eta_{i} \geq 0$, and $\sum_{i} \eta_{i}=\eta \leq 1$ (those considered in [6]), belong to this class, but smooth functions with support within that interval do it as well. Then it is straightforward to check that [11]

$$
n_{0}=-\frac{1}{2} s_{x} \frac{\partial n_{1}}{\partial x}
$$

where $s_{x}=\operatorname{sign} x$. Now, if we consider that

$$
-\ln \left(1-n_{1}\right) \frac{\partial n_{1}}{\partial x}=\frac{\partial \Phi^{(0)}\left(n_{1}\right)}{\partial x}
$$

where 55 $\Phi^{(0)}(\eta)=\eta+(1-\eta) \ln (1-\eta)$, we can check that $F^{\mathrm{ex}}=\int d x \Phi^{(1)}=\Phi^{(0)}\left(n_{1}(0)\right)$, which reduces to the excess free energy of the 0D system for any density of the quasi-0D class.

To check the $2 \mathrm{D}$ to $1 \mathrm{D}$ and $2 \mathrm{D}$ to $0 \mathrm{D}$ dimensional crossovers, let us define the quasi-1D density class as the set of $2 \mathrm{D}$ functions with support in the band $-\sigma / 2<$ $y<\sigma / 2$, and let us redefine the quasi-0D density class as the subset of the quasi-1D class whose members vanish for $x \notin(-\sigma / 2, \sigma / 2)$ (i.e. $2 \mathrm{D}$ functions with support in a square centered at the origin). Let us consider now the $2 \mathrm{D}$ functional (5), and let us put $\mathbf{n}_{1}=\left(n_{1, x}, n_{1, y}\right)$; then $n_{1}^{2}-\mathbf{n}_{1} \cdot \mathbf{n}_{1}=2 n_{1, x} n_{1, y}$. On the other hand, as in the previous case,

$$
n_{0}=-\frac{1}{2} s_{y} \frac{\partial n_{1, x}}{\partial y}, \quad n_{1, y}=-\frac{1}{2} s_{y} \frac{\partial n_{2}}{\partial y}
$$

therefore

$$
\Phi^{(2)}=-\frac{1}{2} s_{y} \frac{\partial}{\partial y}\left[-n_{1, x} \ln \left(1-n_{2}\right)\right],
$$

and then

$$
\begin{aligned}
F^{\mathrm{ex}} & =\int d x d y \Phi^{(2)} \\
& =\int d x \Phi^{(1)}\left(n_{1, x}(x, 0), n_{2}(x, 0)\right),
\end{aligned}
$$

which is the right $2 \mathrm{D}$ to $1 \mathrm{D}$ dimensional crossover. Furthermore, as we have discussed previously, the functional (10) has the right dimensional crossover to 0D for any quasi-0D density.

In both cases, 1D and 2D, the right dimensional crossover arises as a consequence of the fact that for quasi-0D densities the functionals can be obtained as

$$
\begin{aligned}
\Phi^{(1)} & =-\frac{1}{2} s_{x} \frac{\partial \Phi^{(0)}}{\partial x} \\
\Phi^{(2)} & =\left(-\frac{1}{2}\right)^{2} s_{x y} \frac{\partial^{2} \Phi^{(0)}}{\partial y \partial x} .
\end{aligned}
$$


The dimensional crossovers then follow from the direct integration of the derivatives. It can then be inferred that the D-dimensional functional with the right dimensional crossovers will, for quasi-0D densities, be expressed as

$$
\Phi^{(D)}=\left(-\frac{1}{2}\right)^{D} s_{x_{1} \cdots x_{D}} \frac{\partial^{D} \Phi^{(0)}}{\partial x_{D} \cdots \partial x_{1}}
$$

but if we compute the $3 \mathrm{D}$ case, and take into account that

$$
\begin{aligned}
\mathbf{n}_{2} & =\left(-s_{x} \frac{\partial n_{3}}{\partial x},-s_{y} \frac{\partial n_{3}}{\partial y},-s_{z} \frac{\partial n_{3}}{\partial z}\right), \\
\mathbf{n}_{1} & =\left(s_{y z} \frac{\partial^{2} n_{3}}{\partial y \partial z}, s_{z x} \frac{\partial^{2} n_{3}}{\partial z \partial x}, s_{x y} \frac{\partial^{2} n_{3}}{\partial x \partial y}\right), \\
n_{0} & =-s_{x y z} \frac{\partial^{3} n_{3}}{\partial x \partial y \partial z},
\end{aligned}
$$

we find that $\Phi_{1}^{(3)}$ and $\Phi_{2}^{(3)}$ are the same as those in Eq. (2), but $\Phi_{3}^{(3)}$ is given by

$$
\Phi_{3}^{(3)}=\frac{n_{2}^{3}-3 n_{2} \mathbf{n}_{2} \cdot \mathbf{n}_{2}-2 \mathbf{n}_{2} \cdot \mathbf{n}_{2} \cdot \mathbf{n}_{2}}{6\left(1-n_{3}\right)^{2}},
$$

where the notation $\mathbf{v} \cdot \mathbf{v} \cdot \mathbf{v}=v_{x}^{3}+v_{y}^{3}+v_{z}^{3}$ has been introduced. This new expression differs from that of Eq. (2) not only in the numerical coefficients, but also in the appearance of a new term, $\mathbf{n}_{2} \cdot \mathbf{n}_{2} \cdot \mathbf{n}_{2}$, which has no equivalent in HS [2,6]. At first this may seem a spurious term, because it is not even rotationally invariant; on second thoughts, there is no reason why it should be, since the system itself (PHC) is not rotationally invariant either. Such a symmetry is replaced by a discrete group of symmetries which, restricted to the fact that the vector densities $\mathbf{n}_{1}$ and $\mathbf{n}_{2}$ must have positive components, translates into a simple axes-exchange symmetry. The new term obviously has this symmetry. The important thing is that while this new 3D functional has been built to have right dimensional crossovers, the old one does not even fulfill the $3 \mathrm{D}$ to $0 \mathrm{D}$ one $\left(\Phi_{3}^{(3)}\right.$ develops delta cube divergences when the density approaches a delta function, much in the same way as the old version of the HS FMF did [2]). It can be checked that the new functional stabilizes a solid phase, for packing fractions above $\eta \approx 0.3$, by following the standard procedure of parametrizing the density function by a sum of gaussians placed at the lattice sites [1], whereas the divergences in the old functional give rise to a free energy which decreases monotonously as the gaussians shrink.

But to be correct, the new 3D functional should provide the exact second and third virial coefficients in the density expansion of the DCF, for this is the requirement under which FMF are built 2, 4. It is very remarkable that, when applied to the uniform fluid, the new functional provides exactly the same expressions for both the free energy and the DCF as found in Ref. [4] (bear in mind the different normalization of the weighted densities!). Therefore, the new term introduces nothing new for the uniform fluid, but it drastically changes the behavior of the nonuniform fluid.

Formula (13) provides a simple method to obtain the FMF for a D-dimensional PHC fluid; although the formula only holds true for quasi-0D densities, once the functional form has been derived and the scalar, vector, and tensor weighted densities have been identified, it can also be applied to arbitrary density functions [this is precisely the procedure we have followed to obtain the 3D functional (15)]. At this level, Eq. (13) may seem nothing more than a fortunate trick to obtain the functionals; however, a slight manipulation of the equation will provide further insight on the actual meaning of this method.

First of all, let us consider the slightly more general problem of a fluid of D-dimensional parallel hard parallelepipeds (PHP) of edge-lengths $\sigma^{(1)}, \ldots, \sigma^{(D)}$ along the $x_{1}, \ldots, x_{D}$ axes. If we define $\eta(\mathbf{r})=\rho \otimes \omega_{D}(\mathbf{r})$, with

$$
\omega_{D}(\mathbf{r})=\Theta\left(\frac{\sigma^{(1)}}{2}-\left|x_{1}\right|\right) \cdots \Theta\left(\frac{\sigma^{(D)}}{2}-\left|x_{D}\right|\right),
$$

then, the 0D free-energy density functional for this system will be given by

$$
\Phi_{\mathrm{PHP}}^{(0)}=\eta(\mathbf{r})+[1-\eta(\mathbf{r})] \ln [1-\eta(\mathbf{r})] .
$$

Formula (13) still yields the D-dimensional functional for quasi-0D densities, but if we make use of the identity

$$
-\frac{1}{2} s_{x_{j}} \frac{\partial}{\partial x_{j}} \Theta\left(\frac{\sigma^{(j)}}{2}-\left|x_{j}\right|\right)=\frac{\partial}{\partial \sigma^{(j)}} \Theta\left(\frac{\sigma^{(j)}}{2}-\left|x_{j}\right|\right),
$$

Eq. (13) transforms into

$$
\Phi_{\mathrm{PHP}}^{(D)}=\frac{\partial^{D} \Phi_{\mathrm{PHP}}^{(0)}}{\partial \sigma^{(D)} \cdots \partial \sigma^{(1)}},
$$

which is valid not only for quasi-0D densities, but for completely general density functions (because it does not involve derivatives with respect to the coordinates).

Equation (18) has the important consequence that all the thermodynamics of the inhomogeneous Ddimensional fluid is contained in the 0D functional. This result reveals the same fundamental idea recently formulated for HS [6]. In the latter, however, the derivation is not as simple as for the PHP system. An attempt to find an equivalent to Eq. (18) for HS, where the OD functional acts as a generating functional, has just been carried out [12]. In this work, the D-dimensional FMF for HS, in the version of Ref. B], in which all weighted functions are scalar, is given by the action on the $0 \mathrm{D}$ functional of a differential operator with respect to the sphere radius. The result is nevertheless not as satisfactory as for 
the PHP system (for instance, the 3D DCF generates an unphysical delta function at contact). On the other hand, while Eq. (18) emerges here in a very natural way, the differential operator in [12] is introduced heuristically with some free constants which are fitted afterwards by imposing certain constraints (e.g. to match a given equation of state). On the contrary, the functional for $2 \mathrm{D}$ agrees with the simplified version of the one obtained in [6], so that the method seems promising and, in view of what happens for PHP, it deserves further consideration.

Generalizing (18) to mixtures is straightforward; it simply amounts to writing

$$
\Phi_{\mathrm{PHP}}^{(D)}=\sum_{i} \frac{\partial^{D} \Phi_{\mathrm{PHP}}^{(0)}}{\partial \sigma_{i}^{(D)} \cdots \partial \sigma_{i}^{(1)}},
$$

where $\sigma_{i}^{(j)}$ denotes the edge-length along the $x_{j}$ coordinate of species $i$. Of course, now $\eta(\mathbf{r})=\sum_{i} \rho_{i} \otimes \omega_{i}^{(D)}(\mathbf{r})$, where $\omega_{i}^{(D)}$ is given by Eq. (16) with the $\sigma^{(j)}$ s corresponding to species $i$. The functional (19) has a particularly important application to study liquid-crystalline phases, for if we consider the six possible orientations of a parallelepiped as belonging to six different species, the functional (19) can be understood as representing a restricted-orientation, one-species system of hard parallelepipeds. Besides that, the functional may provide some clues to understand the structure of a FMF for freely-orientable, general anisotropic particles.

The derivation we have made here suggests further extensions of the theory. For instance, consider a system of hard parallel cylinders oriented along the $z$ axis. It is clear that the $3 \mathrm{D}$ to $2 \mathrm{D}$ dimensional crossover, eliminating the $z$ coordinate, will transform this system into a system of hard disks. Therefore, if $\Phi_{\mathrm{HS}}^{(2)}$ is a FMF for hard disks (e.g. those of Refs. [6],12]), the corresponding FMF for the cylinders can be derived from $\widetilde{\Phi}_{\mathrm{HS}}^{(2)}$, the same functional as $\Phi_{\mathrm{HS}}^{(2)}$, but with the weights multiplied by $\Theta(L / 2-|z|)$ ( $L$ being the length of the cylinders) and the $2 \mathrm{D}$ density of disks replaced by a $3 \mathrm{D}$ density of cylinders. The derivation will simply be

$$
\Phi_{\mathrm{cyl}}^{(3)}=\frac{\partial \widetilde{\Phi}_{\mathrm{HS}}^{(2)}}{\partial L} .
$$

This functional can immediately be generalized to a mixture of parallel hard cylinders as in (19), and this provides a tool to study analytically the influence of polydispersity in the phase diagram of liquid crystals [13].

To summarize, we have shown how the FMF for PHC presented in [4] can be regularized to have a right dimensional crossover to any smaller dimension. In the derivation we have found the remarkable result that the FMF for PHP, in any dimension $D$, can be obtained by simply deriving with respect to every edge-length the $0 \mathrm{D}$ functional, thus prompting the idea of this functional being a kind of generating functional (an idea already explored for HS [6]12, but which for the present system reaches its clearest and simplest expression). In passing we have obtained the FMF for a mixture of PHP, and suggested how it can be exploited as a restricted-orientation model for liquid crystals. Finally a further extension of the method permits to find a FMF for a mixture of parallel hard cylinders, thus opening the possibility of studying polydispersity in liquid crystals.

We are indebted to Y. Rosenfeld for calling our attention on this problem; to P. Tarazona for illuminating discussions and for keeping us informed of his progress in studying HS; to J.A. White for sending us their preprint, and to B. Mulder for suggesting the extension of the PHP functional to a restricted-orientation model. One of us (J.A.C.) owes much to a discussion with L. Araujo which turned out to be crucial for the development of this work.

[1] See, for instance, the reviews M. Baus, J. Phys.: Cond. Matter 2, 2241 (1990); Fundamentals of Inhomogeneous Fluids, D. Henderson ed. (Dekker, New York, 1992); G. J. Vroege and H. N. W. Lekkerkerker, Rep. Prog. Phys. 55, 1241 (1992); H. Löwen, Phys. Rep. 237, 249 (1994).

[2] Y. Rosenfeld, Phys. Rev. Lett. 63, 980 (1989); see also Y. Rosenfeld, J. Phys.: Cond. Matter 8, 9289 (1996), and references therein.

[3] E. Kierlik and M. L. Rosinberg, Phys. Rev. A 42, 3382 (1990); Phys. Rev. A 44, 5025 (1991).

[4] J. A. Cuesta, Phys. Rev. Lett. 76, 3742 (1996).

[5] Y. Rosenfeld, M. Schmidt, H. Löwen, and P. Tarazona, J. Phys.: Cond. Matter 8, L577 (1996); preprint (1996).

[6] P. Tarazona and Y. Rosenfeld, preprint (1996).

[7] Y. Rosenfeld, Phys. Rev. A 42, 5978 (1990).

[8] Y. Rosenfeld, J. Phys.: Cond. Matter 8, L795 (1996).

[9] And yet they describe accurately the solid phase [1]. But this solid has unphysical properties, such as a negative density of vacancies, or a regular behavior of the free energy at closest packing [5, \&].

[10] M. Dijkstra and D. Frenkel, Phys. Rev. Lett. 72, 298 (1994); M. Dijkstra, D. Frenkel and J.-P. Hansen, J. Chem. Phys. 101, 3179 (1994).

[11] If instead of considering this class of functions we take that of functions highly localized within the same interval, Eq. (6) becomes an asymptotic equality, so that the higher the localization the more accurate the equation.

[12] A. González, J. A. White, and R. Evans, preprint (1996).

[13] A. Stroobants, J. Phys.: Cond. Matter 6, A285 (1994). 